\title{
Wolfgang Rother \\ Die Ringvorlesungen der Privatdozentinnen und Privatdozenten, Titularprofessorinnen und Titularprofessoren der Universität Zürich 1987-2017
}

Zwischen 1987 und 2017 haben die Privatdozentinnen und Privatdozenten, Titularprofessorinnen und Titularprofessoren der Universität Zürich achtzehn Ringvorlesungen gehalten. Die ersten drei Zyklen wurden von den Privatdozierenden der Philosophischen Fakultät bestritten. Nachfolgend werden die Vorlesungen auf der Grundlage der publizierten Beiträge verzeichnet; zuweilen sind die Titel im Inhaltsverzeichnis anders angegeben als vor dem Beitrag selbst - in diesen Fällen notieren wir den Titel gemäss Beitrag. Im Anschluss an die Bibliographie sind jeweils - sofern bekannt die Titel der nicht veröffentlichten Vorlesungen verzeichnet.

\section{[1.] Wintersemester 1987/88}

\section{Angst}

Hans-Jürg Braun, Alexander Schwarz (Hg.): Angst, Zürich, Verlag der Fachvereine an den schweizerischen Hochschulen und Techniken, 1988, (6), 252, (2) Seiten (Zürcher Hochschulforum 13). Vorlesungsreihe der Privatdozenten der Philosophischen Fakultät I der Universität Zürich, Winter 1987/88. Diese Publikation wurde ermöglicht durch Beiträge der Hochschulstiftung und der Kommission «Legat Dr. h.c. Georges Bloch». 
Hans-Jürg Braun: Angst und Existenz: zu Sören Kierkegaards Reflexionen.

Verena Kast: Angst und Angstbewältigung aus psychologischer Sicht.

Peter Seidmann: Weltangst und Traumsymbolik. Zeitgeschichte als Problem der Traumdeutung.

Marie-Claude Hepp-Reymond: Fürchten, flüchten, fechten: zur Neurobiologie der Angst.

Adolf Dittrich: Angst in aussergewöhnlichen Bewusstseinszuständen.

Walter A. Schelling: Psychologische und anthropologische Perspektiven der Angst.

Alexander Schwarz: Keine Angst in der Sprachwissenschaft.

Rudolf Schwarzenbach: Von der Angst des Redners.

Mary Snell-Hornby: «Angst» und «fear» - Wirklichkeit, Wörterbuch, Übersetzung.

Paul Michel: Gestaltungsformen der Angst in der mittelalterlichen deutschen Literatur.

Christoph Eggenberger: Angst und Gottesfurcht im mittelalterlichen Bild.

Peter Grotzer: Die literarische Überwindung der Angst. Anmerkungen zu Paul Nizon und Hermann Burger.

Beatrice Wehrli: Angst und Aufklärung.

Heinrich Mettler und Heinz Lippuner: Angst: wie ihr begegnet wird in Schillers «Wilhelm Tell».

Harro von Senger: Angst in China.

Kurt R. Spillmann: Angst und Sicherheit im Nuklearzeitalter.

Albert A. Stahel: Der Krieg und die Angst.

\section{[2.] Wintersemester 1990/91}

\section{Liebe und Hass}

Peter Grotzer (Hg.): Liebe und Hass, Zürich, Verlag der Fachvereine an den schweizerischen Hochschulen und Techniken, 1991, (6), 234, (4) Seiten (Zürcher Hochschulforum 20). - Vorlesungsreihe der Privatdozenten und Privatdozentinnen der Philosophischen Fakultät I der Universität Zürich, Winter 1990/91. Diese Publikation wurde ermöglicht durch Beiträge der Hochschulstiftung und der Kommission «Legat Dr. h.c. Georges Bloch».

Christoph Eggenberger: Liebe und Hass - Licht und Finsternis - Himmel und Hölle. Bilder und Texte des Mittelalters.

Walter A. Schelling: Liebe und Hass - ein Grundprinzip des Lebens? 
Martin Bircher: Liebe und Hass in Grimmelshausens «Simplicissimus».

Heinrich Mettler: Hass und Liebe in Goethes «Iphigenie» oder das Verhängnis der Vergangenheit und die Eröffnung der Zukunft.

Margret Walter-Schneider: Heinrich von Kleist: «Penthesilea».

Peter Grotzer: Die Liebe als Tragödie bei Racine und Claudel.

Martin E. Schmid: Verunmöglichte Liebe. Alfred Andersch: «Die Rote».

Peter Ackermann: $\mathrm{Zu}$ - und Abneigung im Japanischen.

Georg Kohler: Postmoderne Entfristung. Über die Antiquiertheit von Leidenschaft.

Peter Seidmann: Hassliebe: existentielle Zwiespältigkeit in humananalytischer Sicht.

Beatrice Wehrli: «Du wirst meiner Liebe nicht entgehen». Ein Schlaglicht auf den bürgerlichen Liebesbegriff.

Brigitte Boothe: Liebe und Hass in der psychoanalytischen Therapie.

Albert A. Stahel: Hass und Menschlichkeit im Krieg.

Verena Kast: Die Funktion von Liebe und Hass in Paarbeziehungen.

Bruno Krapf: Erziehung ist nichts als Liebe ... und was daraus werden kann.

\section{[3.] Wintersemester 1993/94}

\section{Dialog}

Thomas Bearth, Thomas Fries, Albert A. Stahel (Hg.): Dialog, Zürich, vdf Hochschulverlag AG an der ETH Zürich, 1994, 258 Seiten (Zürcher Hochschulforum 22). - Vorlesungsreihe der Titularprofessorinnen und Titularprofessoren sowie der Privatdozentinnen und Privatdozenten der Philosophischen Fakultät I der Universität Zürich, Wintersemester 1993/1994. Diese Publikation wurde ermöglicht durch Beiträge der Hochschulstiftung und der Kommission «Legat Dr. h.c. Georges Bloch». - In memoriam Peter Grotzer (1933-1992).

François Höpflinger: Dialog zwischen den Generationen einer Vier-GenerationenGesellschaft.

Bruno Krapf: Ich kann Dich einfach nicht verstehen! oder: Zur Psychologie der Gesprächsführung.

Margret Walter-Schneider: Ein Fontane-Dialog.

Alexander Schwarz: Was Hildebrand und Hadubrand einander uns was sie uns zu sagen haben.

German Ritz: Aufbruch in eine mehrstimmige Welt. Entwicklung zur Postmoderne in der zeitgenössischen polnischen Lyrik. 
Thomas Fries: Hunger, Politik und Literatur: Ein Dialog am Vorabend der

Französischen Revolution (Galiani: Dialogues sur le commerce des bleds)

Klaus Weimar: Lesen: zu sich selbst sprechen in fremdem Namen.

Elizabeth Couper-Kuhlen: Takt im Dialog. Über das zeitliche Raster des «Miteinanderredens).

Thomas Bearth: «Tut's weh?». - «Nein.»- «Wo?» Sprachlogik und Dia-Logik. Rudolf Schwarzenbach: «Ds Wort isch fry!» Landsgemeindedebatten zwischen Meinungskampf und Ritual.

Harro von Senger: Probleme beim Dialog zwischen Europa und China.

Albert A. Stahel: Krieg oder Dialog im ehemaligen Jugoslawien (Stand 28. Februar 1994).

Christian Doelker: Dialog im und mit dem Fernsehen.

Christoph Eggenberger: Das Dialogbild - der Dialog mit dem Bild.

Carlo Moos: Als Historiker im Gespräch mit der Vergangenheit.

\section{[4./1.] Wintersemester 2001/02 Brüche, Torsi, Unvollendetes}

Kurt Schärer, Erwin Sonderegger (Hg.): Brüche, Torsi, Unvollendetes. Über das Fragmentarische in Leben, Kunst und Wissenschaft, Zürich, Chronos, 2004, 259 Seiten. - Der vorliegende Sammelband umfasst die Vorträge einer im Wintersemester 2001/02 von Privatdozentinnen und Privatdozenten der Universität Zürich durchgeführten Ringvorlesung. Für die finanzielle Unterstützung dieser Publikation danken wir der Kommission «Legat Dr. h.c. Georges Bloch» und der Hochschulstiftung der Universität Zürich.

\section{Welt und Geschichte}

Klaus Felix Kaiser: Mit bruchstückhaften Funden zu einer Klimareihe mit abrupten Einbrüchen.

Philippe Della Casa: Mut zum Unvollendeten. Vom täglichen Umgang mit den Bruchstücken der Vergangenheit.

\section{Literatur}

Michael Andermatt: Mensch in Stücken. Zum Motiv des künstlichen Menschen im Erzählen der europäischen Romantik.

Margret Walter-Schneider: «Wir brauchten Silhouettenstürmer». Über den Ganzheiten-Verächter Jean Paul.

Beatrice Wehrli: Bruch-Stücke. Vom Rausch der Leidenschaft in Kleists «Penthesilea».

Kurt Schärer: Vom schwierigen Umgang mit dem Bruchstückhaften. 


\section{Kunst}

Christoph Eggenberger: Non finito. Unvollendete Bilderhandschriften.

Dorothea Baumann: Konzertsäle und Opernhäuser des 20. Jahrhunderts im Spannungsfeld zwischen Umbruch und Tradition.

\section{Wissen}

Guerino Mazzola: Vom Bruch mit der Disziplin zum Zusammenbruch des Wissens.

Erwin Sonderegger: Das Phänomen der grundsätzlichen Unvollständigkeit.

Aldo Lanfranconi: Nochmals: das Erhabene. $\mathrm{Zu}$ einem traditionellen Konzept des «ästhetisch» Unvollendeten.

\section{Leben}

Jürg Berthold: Bruchloses Glück? Moralphilosophische Überlegungen zum gelingenden Leben.

Martin Sieber: Brüche im Verhältnis von Freiheit und Abhängigkeit. Fallbeispiele zum Konsum von Alkohol.

Markus Huppenbauer: Der Riss, der das Universum durchzieht. Schöpfungstheologische Reflexionen über den Stand der Dinge.

\section{[5./2.] Wintersemester 2003/04}

\section{Königswege, Labyrinthe, Sackgassen}

Kurt Schärer (Hg.): Königswege, Labyrinthe, Sackgassen. Über Formen und Methoden des Denkens, Handelns und Gestaltens, Zürich, Chronos, 2004, 277, (1) Seiten. - Der vorliegende Sammelband umfasst die Vorträge einer im Wintersemester 2003/ 2004 von Privatdozentinnen und Privatdozenten an der Universität Zürich durchgeführten Ringvorlesung. Für die finanzielle Unterstützung dieser Publikation danken wir der Hochschulstiftung, dem Rektor und der Vereinigung der Privatdozenten der Universität Zürich sowie dem Zürcher Universitätsverein.

\section{Philosophie und Theologie}

Marco Baschera: Wege und Methoden. Gedankengänge zur Beziehung von Gehen und Denken.

Jürg Berthold: «Wer denken will, muss sich fragen.» Zu einem Königsweg der Philosophie.

Markus Huppenbauer: Ethik und Rhetorik. Warum es einfacher ist, mit einem Cadillac durch New York zu fahren als wissenschaftliche Ethik zu treiben. 
Jan Bauke: Die Wissenschaftsgläubigkeit der Theologie. Heilspfad oder Holzweg?

\section{Sprache und Literatur}

Karin Stüber: Die Entzifferung des Hethitischen: Irrwege und Durchbruch.

Beatrice Wehrli: Von Königswegen und Königinnen: Ingeborg Bachmann und Elfriede Jelinek.

Maya Schärer-Nussberger: Königsweg und Labyrinth. Natur versus Zivilisation im venezolanischen Roman.

Kurt Schärer: Textlabyrinthe beim französischen Romancier Julien Gracq.

\section{Wissenschaft und Kritik}

Sebastian Bott: Tells Bogen, Goethes Nase, Mesmers Wanne. Scharlatanerie und Wissenschaft in Zürich um 1800.

Guerino Mazzola: Das Labyrinth der Schizophrenie, die Musik und das digitale Zeitalter.

Daniel Strassberg: E pluribus unum. Über die verborgene Theologie der Psychoanalyse.

Stefanie Stadler Elmer: «Wo man singt, da lass dich ruhig nieder ...». Erziehung und Verführung durch Lieder.

Albert A. Stahel: Die Kriegführung der USA: eine politische Sackgasse?

Martin Sieber: Wege und Irrwege der Alkoholismusforschung. Zur Eugenikdebatte und zur Rolle der Psychiatrie.

\section{[6./3.] Wintersemester 2004/05}

\section{Erbe, Erbschaft, Vererbung}

Werner M. Egli, Kurt Schärer (Hg.): Erbe, Erbschaft, Vererbung, Zürich, Chronos, 2005, 263 Seiten. - Der vorliegende Sammelband umfasst Vorträge einer im Wintersemester 2004/ 05 von Privatdozentinnen und Privatdozenten an der Universität Zürich gehaltenen Ringvorlesung. Für die finanzielle Unterstützung dieser Publikation danken wir der Hochschulstiftung der Universität Zürich, dem Zürcher Universitätsverein und der KENDRIS private AG, Zürich.

\section{Eigentum}

Peter Breitschmid: Das Erbrecht des 19. im 21. Jahrhundert. Der Konflikt zwischen Status, Realbeziehung und erblasserischer Freiheit.

Werner Egli: Erben im Kulturvergleich - oder warum die Bauern so anders sind. 
11 Wolfgang Rother: Ringvorlesungen 1987-2017

Willemijn de Jong: «Unsere Stärke liegt in unserem eigenen Haus». Matrilinearität und soziale Sicherheit in Südindien.

\section{Erbanlagen}

Martin Schwyzer: Herpesviren als Erbschleicher.

Béatrice Ziegler: «Der Mensch gilt, was sein Erbgut wert ist». Eugenik und nationale Solidargemeinschaft.

Hans-Konrad Schmutz: «Völker wechseln - Rassen bleiben». Rassendiskurs und Nationalgeschichte im späten 19. Jahrhundert.

Ursula Bähler: Vererbung als Romanprinzip: «Les Rougon-Macquart von Emile Zola.

\section{Tradition}

Jürg Berthold: «Das Matterhorn - vom Gorner Grat aus betrachtet». Über Tradition nach einer Bemerkung Adornos.

Sebastian Bott: Das Erbe der Tugend. Zur politischen Sprache Zürichs im 18./19. Jahrhundert.

Rudolf Schwarzenbach: Vaterland und Muttersprache. Traditionen sprachlicher Identifikation.

Maya Schärer-Nussberger: Lateinamerika zwischen Tradition und Utopie.

Hinterlassenschaft

Wolfgang F. Kersten: «Für die Nachlass-Sammlung bestimmt». Strategien zur Gewinnung unsterblichen Ruhms. Von Dürer über Klee bis Beuys.

Kurt Schärer: Die Erbverweigerung bei André Gide.

\section{[7./4.] Wintersemester 2005/06}

\section{Spuren}

Kurt Schärer, Karin Stüber (Hg.): Spuren, Zürich, Chronos, 2006, 277, (1) Seiten. - Der vorliegende Sammelband umfasst Vorträge einer im Wintersemester 2005/06 von Privatdozentinnen und Privatdozenten an der Universität Zürich gehaltenen Ringvorlesung. Für die finanzielle Unterstützung dieser Publikation danken wir der Hochschulstiftung und der Privatdozenten-Stiftung der Universität Zürich sowie dem Zürcher Universitätsverein.

\section{Philosophie und Theologie}

Jürg Berthold: Die Spur des Menschen, der Mensch als Spur.

Sebastian Bott: Spuren des Göttlichen in der Natur. Überlegungen zur Mensch/

Tier-Differenz im 18. Jahrhundert. 
Jan Bauke: Gottes Spur(en). Zur philosophischen Dekonstruktion und theologischen Renaissance eines vielschichtigen Topos.

Marco Baschera: Das Sichtbare als Spur des Unsichtbaren.

\section{Geschichte}

Karin Stüber: Sprachliche Spuren der Kelten in der Schweiz.

J. Jürgen Seidel: Ein Nonkonformist als Wohltäter. Religiöse und soziale Spuren Carl Joseph von Campagnes (1751-1833) im Zürcher Oberland.

Albert A. Stahel: Das kriegsverwüstete Afghanistan. Spuren der Vergangenheit - und die Zukunft?

\section{Literatur}

Maya Schärer-Nussberger: Das Wort als Spur und Zeugnis. Zwischen Realität und Fiktion.

Beatrice Wehrli: Gotthold Ephraim Lessing: Wahrheit als Spur im Begriff des Irrtums.

Michael Andermatt: Junggesellenmaschinen. Spuren romantischer Liebe in der ästhetischen Moderne. Von F. Schlegel und E. T. A. Hoffmann zu G. Meyrink und M. Duchamp.

Kurt Schärer: Eine Poetik der Spuren: Der französische Symbolismus.

Christina Vogel: Spuren lesen als Aufgabe der modernen Lyrik. Philippe Jaccottet und Yves Bonnefoy.

Medizin und Wirtschaftswissenschaft

Marek Fischer: Spuren des HI-Virus unter antiretroviraler Therapie.

Alfred Metzler: Genetische Spuren der Polioviren und die Hoffnung auf eine Welt ohne Kinderlähmung.

André C. Wohlgemuth: Dürfen Unternehmensberater Spuren hinterlassen?

\section{[8./5.] Wintersemester 2006/07}

\section{Spurensuche - Spurenlese}

Kurt Schärer (Hg.): Spuren lesen, Zürich, Chronos, 2007, 235 Seiten. - Der vorliegende Sammelband enthält Vorträge einer im Wintersemester 2006/2007 von Privatdozenten und Privatdozentinnen an der Universität Zürich gehaltenen Ringvorlesung. Für die finanzielle Unterstützung dieser Publikation danken wir der Hochschulstiftung der Universität Zürich, dem Zürcher Universitätsverein sowie Herrn Toni Hauswirth.

\section{Philosophie}

Guerino Mazzola: Das Denken - Asservatenkammer erstarrter Gesten?

Marco Baschera: Die Stimme als Spur des Körpers in der Sprache. 
Jürg Berthold: Wissenschaftliches Denken als kollektives Spurenlesen? Ludwik Flecks Überlegungen zur «Entstehung einer wissenschaftlichen Tatsache».

Literatur- und Sprachwissenschaft

Maya Schärer-Nussberger: Im Spiel der Spuren: Dichtung und Intertextualität. Kurt Schärer: Maurice Maeterlinck - eine Dramaturgie der Spuren.

Karin Stüber: Die Stellung der Frau: Spuren indogermanischer Gesellschaftsordnung in der Sprache.

Geschichte und Ethnologie

Sebastian Bott: «Von Europa aber weiss offenbar niemand etwas Genaues ...». Eine historische Spurenlese zum Europäischen und zu seiner pädagogischen Relevanz.

Willemijn de Jong: Konfliktspuren und Rituale in Ostindonesien.

Afghanistan heute. Spuren der Hoffnung?

Albert A. Stahel: Das Bildungswesen.

Beat Nick: Das Gesundheitswesen. Vergängliche Spuren.

Enrique Steiger: Wunsch und Wirklichkeit im Gesundheitswesen.

\section{Medizin}

Stefan Büchi: «Leiden vergeht - gelitten haben nie». Seelische Spuren von Krankheit und Verlust.

Markus Schneemann: Thermometer: Spurensicherung am menschlichen Körper. Wann ist Fieber?

Ralf W. Baumgartner: Streifung - Vorbote einer Katastrophe.

Hubert John: Die Suche nach der optimalen Prostatakrebsoperation. Führt die Spur zur roboterassistierten radikalen Prostatektomie?

\section{Nicht veröffentlichte Vorlesungen}

Martha Koukou: Spuren der Kultur und die Entstehung der Individualität: eine hirnphysiologische Betrachtung.

Rolf Streuli: Spuren, die zur Diagnose von Krankheiten führen.

Hans C. Steinert: Mit Stoffwechselspionen auf der Suche nach Krebszellen.

Peter Schmid-Grendelmeier: Auf den Spuren von Mythen, Massai und Molekülen: Pilzallergien beim Ekzem.

\section{[9./6.] Herbstsemester 2007}

\section{Intimität}

Ingrid Tomkowiak, Werner Egli (Hg.): Intimität, Zürich, Chronos, 2008, 284 Seiten. - Redaktionelle Mitarbeit: Aleta-Amirée von Holzen. - Der vorliegende Sammelband enthält die Vorträge einer im Herbstsemester 2007 von Privatdozenten und Privatdozentinnen, 
Titularprofessorinnen und Titularprofessoren an der Universität Zürich gehaltenen Ringvorlesung. Gefördert mit Mitteln der Hochschulstiftung Zürich und des Zürcher Universitätsverein (ZUNIV).

\section{Öffentlichkeit und Privatheit}

Tomas Poledna: Technische Entwicklungen und Datenschutz: Ein Hase-undIgel-Rennen?

Johanna Rolshoven: Die Wegweisung. Die Züchtigung des Anstössigen oder: Die europäische Stadt als Ort der Sauberkeit, Ordnung und Sicherheit.

Beat Fux: Die Intimisierung des öffentlichen Raumes. Über das Zusammenspiel von Selbstdarstellung, sozialer Schliessung und Integration.

Urs Dahinden: Intimität und mediale Öffentlichkeit: Kein Gegensatz mehr?

Daniel Süss, Gregor Waller: Erziehungsberatung als öffentliches Spektakel? Analysen zur Fernseh-Super-Nanny.

\section{Körperlichkeit, Verwandtschaft}

Christoph Eggenberger: Die Intimität der Maria. Das Verkündigungsbild im Turin-Mailänder Stundenbuch.

Willemijn de Jong: Intimität auf dem Prüfstand. Neue Verwandtschaftsverhältnisse durch assistierte Reproduktionstechnologien.

Gabriella Milos: Modernes Schönheitsideal und Essstörungen. Wie die Manipulation des eigenen Körpers krank machen kann.

Vertrautheit, Freundschaft, Liebe

Heinz Käufeler: Vertrauen, Vertrautheit, Vertraulichkeit. Über den Umgang mit Intimität in der Ethnologie.

Jürg Berthold: Intimus. Derridas Philosophie der Freundschaft.

Albert A. Stahel: Intimität und Freundschaft zwischen Männern in Kriegssituationen. Meine Erfahrungen in Afghanistan.

Thomas Fries: «Ich will dir alles erzählen». Intimität zwischen Frau und Mann in Gottfried Kellers Sinngedicht und Arthur Schnitzlers Traumnovelle.

Astrid Riehl-Emde: Intimität in der Paarbeziehung - ein Prozess voller Risiken

Innerlichkeit

Dorothea Baumann: Musik von innen her empfinden und verstehen. Einsichten aus Susanne K. Langers Kunstphilosophie.

\section{[10./7.] Herbstsemester 2008}

\section{Sinne}

Werner M. Egli, Ingrid Tomkowiak (Hg.): Sinne, Zürich, Chronos, 2010, 229 Seiten. - Redaktionelle Mitarbeit: Helene Mühlestein. Der vorliegende Sammelband enthält Beiträge der Ringvorlesung 
der Privatdozierenden an der Universität Zürich im Herbstsemester 2008. Gefördert mit Mitteln der Hochschulstiftung Zürich und des Zürcher Universitätsverein (ZUNIV).

Werner M. Egli: Einleitung. Sinn, Sinne, Sinnessysteme - Bedeutungen, Perspektiven, Themen.

Erwin Sonderegger: Ohne Sinne kein Sinn und keine Sinne ohne Sinn.

Markus Huppenbauer: Sinneswahrnehmung und Moral. Phänomenologische und theologische Überlegungen zur Ethik.

Oliver Zerbe: Die molekularen Mechanismen des Sehens.

Wolfgang Kersten: Blicke aus Bildern. Fallstudien zu Franz von Lenbach, Candida Höfer und Franz Gertsch.

Stefanie Stadler Elmer: Hören - eine entwicklungspsychologische Perspektive.

Stephan Schmid: Die Wahrnehmung der Sprachlaute. Wissenschaftsgeschichtliche Streiflichter auf Phonetik und Phonologie.

Werner M. Egli: Bambus als Achse des Sinnessystems in einer indigenen Psychologie des Himalajas. Die «Sunuwar» und ihr Sinn für Kommunikation.

Dorothee Rippmann: Körper und Sinne in der Kochkunst. Konzepte mittelalterlicher Nahrungsmitteldiätetik.

J. Jürgen Seidel: Mit Herzen, Mund und Händen. Liedkultur und Biografie im christlichen Kontext.

Hubert John: Patient und technikorientierte Medizin am Beispiel der Urologie. Verlieren wir unsere Sinne?

Michael Hässig: Die Sinne des Tieres. Ein neuer Ansatz im Tierschutz.

Nicht veröffentlichte Vorlesungen

Albert A. Stahel: Sinnigkeit und Sinnlichkeit auf der Seidenstrasse.

\section{[11. / 8.] Herbstsemester 2009}

\section{Berge}

Werner M. Egli, Ingrid Tomkowiak (Hg.): Berge, Zürich, Chronos, 2011, 160 Seiten. - Redaktionelle Mitarbeit: Barbara Loepfe. Der vorliegende Sammelband enthält Beiträge der Ringvorlesung der Privatdozierenden an der Universität Zürich im Herbstsemester 2009. Gefördert mit Mitteln der Hochschulstiftung Zürich und des Zürcher Universitätsverein (ZUNIV). 
Werner M. Egli, Ingrid Tomkowiak: Berge als extreme Landschaften, Sinnbilder und Perspektiven. Aspekte eines paradoxen Faszinosums.

Norman Backhaus: Alpensichten. Ein Landschaftsmodell zur Konfliktvermeidung.

Roland Altenburger: Der heilige Gipfel des Ostens. Staatsritual, Pilgerschaft und Besichtigungstour auf dem Berg Tai.

Christoph Eggenberger: Sinai, Tabor, Zion. Der Berg in der Malerei.

Michael Andermatt: Transformationen des Sakralen. Zur Literarisierung des Berges von der Aufklärung bis zur Gegenwart.

Daria Pezzoli-Olgiati: Alpenbilder. Der Berg im Spiegel religiöser Praxis am Beispiel von Votivbildern aus dem 19. Jahrhundert.

Werner M. Egli: Woher kommen die Findlinge? Wie die frühe Hochgebirgsforschung zur Erfindung der «primitiven Gesellschaft» beitrug.

Hans-Konrad Schmutz: Homo alpinus oder die vermessene Nation.

Josette Baer: Das Tatra-Gebirge als Symbol der slowakischen nationalen und politischen Identität.

Conradin A. Burga, Esther Frei, Romedi Reinalter, Gian-Reto Walther. Wenn Pflanzen in die Höhe wandern. Beobachtungen zur Flora von Berggipfeln der alpinen Stufe im Engadin.

\section{[12. / 9.] Herbstsemester 2011}

\section{Körper}

Josette Baer, Wolfgang Rother (Hg.): Körper. Aspekte der Körperlichkeit in Medizin und Kulturwissenschaften, Basel, Schwabe, 2012, 198, (2) Seiten (Schwabe interdisziplinär 1). - [Ringvorlesung der Privatdozierenden, Titularprofessorinnen und Titularprofessoren der Universität Zürich, Herbstsemester 2011]. Publiziert mit Unterstützung der Hochschulstiftung der Universität Zürich und des Zürcher Universitätsvereins.

Wolfgang Rother: Einleitung. Aspekte einer Metaphysik des Körpers.

Peter Biro: Warum sich Atemweg und Magen-Darm-Trakt kreuzen und für

Kopfschmerzen bei den Anästhesisten sorgen.

Stephan Vavricka: Trägt die Darmflora zu unserem Wohlbefinden bei?

Iris Ritzmann: Lesarten des Körpers im Zeitalter der Eugenik.

Barbara Lay: Die Sorge um Figur und Gewicht. Welchen Einfluss haben gesellschaftliche Entwicklungen auf das Körperbild? 
Werner M. Egli: Körper, Trance und freie Rede. Schamanismus und Ahnenkult in Ost-Nepal.

J. Jürgen Seidel: Religion und Erotik. Von der Lust und Last des Körpers.

Jürg Berthold: Den eigenen Körper denken. Überlegungen zu Jean-Luc Nancy.

Christina Vogel: «Je suis par moments dans le creux de ma main». Paul Valérys Theorie der Hand.

Josette Baer: Živena - die helfende weibliche Hand? Zur Lage der Frauen in der Slowakei vor dem Ersten Weltkrieg.

Wolfgang Rother: Der nackte Körper und die Frage nach der Wahrheit. Bemerkungen zu Degas, Nietzsche und Heidegger.

\section{Nicht veröffentlichte Vorlesungen}

Stephan Schmid: Sprechorgane.

Radu Tutuian: Laktoseintoleranz - eine aus der Luft gegriffene Diagnose.

Karim Eid: Arthrose: Löst der biologische Gelenksersatz bald die Prothese ab? Christina Ljungberg: The Body as Site in the Works of Siri Hustvedt, Paul Auster and Sophie Calle.

Peter Brugger: Phantome: Variationen ausserkörperlicher Empfindungen.

Gabriella Milos: Körper und Essstörungen.

\section{[13./10.] Herbstsemester 2012}

\section{Geld}

Josette Baer, Wolfgang Rother (Hg.): Geld. Philosophische, literaturwissenschaftliche und ökonomische Perspektiven, Basel, Schwabe, 2013, 250, (2) Seiten (Schwabe interdisziplinär 2). - [Ringvorlesung der Privatdozierenden, Titularprofessorinnen und Titularprofessoren der Universität Zürich, Herbstsemester 2012]. Publiziert mit Unterstützung der Hochschulstiftung der Universität Zürich, des Zürcher Universitätsvereins und der Vereinigung der Privatdozierenden der Universität Zürich.

Ursula Pia Jauch: Von Geld und Kathedralen. Philosophische Gedanken über ein Lebensmittel.

Peter Seele: Geld als anthropogene Religion.

Ulrike Zeuch: Geld und Macht in Faust II.

Simone Müller: Das Geld in der japanischen Literatur. Aspekte von Haben und Sein. 
Patricia M. Schiess Rütimann: Politikfinanzierung in der Schweiz. Der Gesetzgeber ist gefordert.

Josette Baer: Karel Havlíček: Warum bin ich ein Bürger? Steuerlast und Korruption im Böhmen des 19. Jahrhunderts.

Wolfgang Rother: Die Wirtschaftslehre Johann Adam Völlingers. Zur Aktualität eines vergessenen Ökonomen der deutschen Spätaufklärung.

Ulrike Stefani und Benjamin Heß: Abschlussprüfung nach der Finanzkrise.

Rolf Oppliger: Geld im digitalen Zeitalter. Eine Standortbestimmung.

Matthias Schwenkglenks: Geld und Medizin. Ökonomische Spannungsfelder im Gesundheitswesen.

Eli Alon: Gesundheitsökonomische Aspekte der Behandlung chronischer Schmerzen.

\section{Nicht veröffentlichte Vorlesungen}

Peter Schmid-Grendelmeier: Lots of skin - and little money: skin diseases in tropical areas with limited resources.

Clemens H. Cap: Bitcoin - das digitale Open-Source-Geld des Internet-Zeitalters.

\section{[14./11.] Herbstsemester 2013}

\section{Arbeit}

Josette Baer, Wolfgang Rother (Hg.): Arbeit. Philosophische, juristische und kulturwissenschaftliche Studien, Basel, Schwabe, 2014, 212, (4) Seiten (Schwabe interdisziplinär 4). - [Ringvorlesung der Privatdozierenden, Titularprofessorinnen und Titularprofessoren der Universität Zürich, Herbstsemester 2013]. Publiziert mit Unterstützung der Hochschulstiftung der Universität Zürich, des Zürcher Universitätsvereins und der Vereinigung der Privatdozierenden der Universität Zürich.

Urs Marti-Brander: Arbeit - (k)ein Thema der politischen Philosophie?

Wolfgang Rother: Jenseits von Langeweile und Müßiggang. Bemerkungen zu Kierkegaards Begriff der Arbeit.

Simone Müller: Der Intellektuelle als Müßiggänger in der japanischen Literatur des frühen 20. Jahrhunderts. Natsume Sōseki und die Gruppe der Yoyūha. Ulrike Zeuch: Von Müßiggängern, Nichtstuern und anderem Gesindel. Zum Lob der Faulheit in der Literatur.

Harald Bärtschi und Andreas Lienhard: «Jeder Arbeiter ist seines Lohnes wert». Rechtliche Schranken der Lohnfestlegung. 
Werner M. Egli: Kinderarbeit - Beurteilung und Verurteilung in kulturvergleichender Perspektive.

Patricia M. Schiess Rütimann: Streik - Offene Fragen im Zeitalter der Informations- und Dienstleistungsgesellschaft.

Eli Alon und Daniel Richter: Schmerzen und Arbeitsunfähigkeit. Medizinische und forensische Aspekte.

Josette Baer: Ein Catch-22? Die slowakischen Sozialdemokraten zwischen nationaler Identität und internationaler Arbeitersolidarität (1905-1918).

\section{Nicht veröffentlichte Vorlesungen}

Ursula Pia Jauch: Notizen aus dem Leben einer thrakischen Magd, oder: Arbeitet eine Philosophin?

Eckhard Deschler Erb: Homo Faber. Zwischen Heimarbeit und Manufakturwesen. Das Handwerk in den römischen Nordwestprovinzen.

Stephan Vavricka: Die menschliche Verdauung: die tägliche Schwerarbeit im Körper.

Alexandra Kleihues: Poetik der Arbeit nach 1945.

\section{[15./ 12.] Herbstsemester 2014}

\section{Vertrauen}

Josette Baer, Wolfgang Rother (Hg.): Vertrauen, Basel, Schwabe, 2015, 231, (1) Seiten (Schwabe interdisziplinär 7). - [Ringvorlesung der Privatdozierenden, Titularprofessorinnen und Titularprofessoren der Universität Zürich, Herbstsemester 2014]. Publiziert mit Unterstützung der Hochschulstiftung der Universität Zürich, des Zürcher Universitätsvereins und der Vereinigung der Privatdozierenden der Universität Zürich.

Wolfgang Rother: Vertrauen als Existenzial. Einige vorläufige Notizen. Matthias Neugebauer: (Erschüttertes) Gottvertrauen? Von der Alltäglichkeit und Unalltäglichkeit eines religiösen Grundphänomens.

Dominique Kuenzle: Weiß er, wovon er spricht? Vertrauen in das Wissen anderer.

Ulrike Zeuch: « ... aber die Dichter lügen zu viel.» Zum Vertrauen in die Wahrheit der Literatur.

Michael Andermatt: Letales Glück. Vertrauen in den deutschen Idealismus. Gottfried Kellers Roman Der grüne Heinrich (1. Fassung 1854/55).

Clemens H. Cap: Kann man einem Computer vertrauen? 
Michael Hässig: Kennen Tiere Vertrauen?

Iris Ritzmann: Vertrauen als Mittel zur Patientenbindung. Historische Blicke auf eine ärztliche Strategie.

Kyrill Schwegler und Eli Alon: Vertrauen in der Arzt-Patienten-Beziehung.

Josette Baer: Vertrauen ist nichts, Macht ist alles. Gustáv Husák - Versuch eines politischen Psychogramms.

Peter Seele und Chr. Lucas Zapf: Berechnendes Vertrauen in den blinden Glauben an den Markt. Eine Medienanalyse.

André C. Wohlgemuth: Vertrauensverlust in der Wirtschaft?

\section{Nicht veröffentlichte Vorlesungen}

Stephan Vavricka: Kommunikation über Risiken als vertrauensbildende Massnahme - die Sicht eines Arztes.

Antoinette Weibel: Vertrauen und Kontrolle.

\section{[16./ 13.] Herbstsemester 2015 \\ Verbrechen und Strafe}

Josette Baer, Wolfgang Rother (Hg.): Verbrechen und Strafe, Basel, Colmena, 2017, 216 Seiten (Colmena Perspektiven I). - [Ringvorlesung der Privatdozierenden, Titularprofessorinnen und Titularprofessoren der Universität Zürich, Herbstsemester 2015]. Publiziert mit Unterstützung der Hochschulstiftung der Universität Zürich, der Privatdozenten-Stiftung der Universität Zürich, des Zürcher Universitätsvereins und der Vereinigung der Privatdozierenden der Universität Zürich.

Frank Ueberschaer: Auf der Suche nach der angemessenen Strafe. Perspektiven alttestamentlicher Literatur.

Marc Winter: Die Todesstrafe im kaiserlichen China und ihre Wahrnehmung im Westen.

Jan-Andrea Bernhard: Protestantische Inquisition? Die Bündner Synode in ihrem Auftrag «zu wysen, warnen, vermanen unnd straffen» (15372016).

Barbara von Orelli-Messerli: Ornament und Verbrechen. Adolf Loos' kontroverser Vortrag.

Erich Bryner: Fjodor Dostojewskis Roman Verbrechen und Strafe.

Josette Baer: Massenmord und Todesstrafe im kommunistischen Prag. Der Fall Olga Hepnarová. 
21 Wolfgang Rother: Ringvorlesungen 1987-2017

Ulrike Zeuch: «Durften sie schiessen?» Maueropfer in der Literatur der DDR und der Nachwendezeit.

Wolfgang Rother: Ein Beitrag zur Humanisierung des Strafprozesses: Johann Christian Gottlieb Schaumanns Ideen zu einer Kriminalpsychologie (1792).

Jürg Berthold: ... oder Verzeihen. Überlegungen zu einer Philosophie der Vergebung.

\section{Nicht veröffentlichte Vorlesungen}

Simone Müller: Von der Strafbarkeit des Übersetzens: der Fall Lady Chatterley in Japan.

Matthias Barton: Die rechtlich-ethische Verantwortung von Hochschulen und Politik am Beispiel von Andreas Grüntzig (1939-1985).

Christine Bartsch: Vom Verbrechen zur Strafe - CSI Zürich.

Urs Marti-Brander: Foucault - die Macht und die Frage nach der Legitimität des Strafens.

\section{[17./ 14.] Herbstsemester 2016 \\ Terror, Angst und Schrecken}

Josette Baer, Wolfgang Rother (Hg.): Terror, Basel, Colmena, 2017, 204 Seiten (Colmena Perspektiven II). - [Ringvorlesung der Privatdozierenden, Titularprofessorinnen und Titularprofessoren der Universität Zürich, Herbstsemester 2016]. Publiziert mit Unterstützung der Vereinigung der Privatdozierenden der Universität Zürich, der Hochschulstiftung der Universität Zürich und des Zürcher Universitätsvereins.

Wolfgang Rother: «Die absolute Freiheit und der Schrecken». Zur Dialektik der Revolution im Anschluss an Hegel.

Ulrike Zeuch: Terror in der Literatur des 21. Jahrhunderts.

Erich Bryner: Revolution und Terror. Nihilismus, Anarchismus und Marxismus im Russland des 19. Jahrhunderts.

Christian Koller: Fake News im Weltenbrand. Gewalt und Emotionen in der Propaganda des Ersten Weltkriegs.

Michael Hässig: Terror und biologische Waffen. Historische und kulturelle Perspektiven.

Udo Döbbeling: Gewalt gebiert Gewalt. Beeinflussen Gene die Gewalttätigkeit und Gewalttätigkeit unsere Gene? 
Josette Baer: Nach dem Terror die Angst. Der psychologische Alltag im Kommunismus.

Henriette Haas: Die gefährlichsten Sätze der Welt. Wie junge Menschen dazu verführt werden, sich gewalttätigen Gruppen anzuschliessen und in den Krieg zu ziehen.

Barbara von Orelli-Messerli: Sünden, Angst und Schrecken in Hieronymus Boschs malerischem Universum.

Werner M. Egli: Always connected. Ist Terror der Normalzustand jeder oralen Gesellschaft?

\section{Nicht veröffentlichte Vorlesungen}

Margaret Jacques: Gott sehen: Die erschreckende Sicht der göttlichen Herrlichkeit in Texten und Bildern des alten Mesopotamien.

Jan-Andrea Bernhard: Das Ende des Stephansreiches und der Mythos von der Schreckensherrschaft der Türken.

Prasenjit Saha: Der Plural von «Anekdote» heisst nicht «Statistik».

Urs Marti-Brander: Die Schrecken des Bürgerkriegs in der griechischen Antike. Stefan Hegemann: Teufelskreis Schwindel - Angst.

\section{[18./ 15.] Herbstsemester 2017}

\section{Lust und Leiden}

\section{Josette Baer, Wolfgang Rother (Hg.): Lust und Leiden}

Wolfgang Rother: Zur Ontologie von Lust und Leiden.

Ulrike Zeuch: Goethes Trilogie der Leidenschaft.

Barbara von Orelli-Messerli: Otto Dix - der Künstler, der alles sehen wollte.

Der Blick auf Leiden und Lust in der Neuen Sachlichkeit.

Christian Koller: Leiden und «Lust» in der Fremdenlegion.

Erich Bryner: Lust und Leiden in Tolstojs Anna Karenina.

Josette Baer: Politik und Mode in der sozialistischen Tschechoslowakei.

Marc Winter: Lust und Schmerz als sexuelle Konzepte? Warum es im vormodernen China keinen Sadomasochismus gab.

Hubert John und Philipp Maletzki: Vom Frust zur Lust in der Urologie. Harninkontinenz und erektile Dysfunktion.

\section{Nicht veröffentlichte Vorlesungen}

Iris Ritzmann: Wenn die Lust zum Leid wird - medizinhistorische Überlegungen zu Pathologisierung von Sexualität.

Stephan Vavricka: Fasten: die Grenzerfahrung zwischen Leiden und Lust. 
23 Wolfgang Rother: Ringvorlesungen 1987-2017

Wolfgang Rother: Lust, Leiden, Schmerz, Krankheit und Tod. Existenzphilosophische Perspektiven.

Eli Alon: Mit Lust gegen das Leiden: Cannabis für die Schmerzbehandlung Lust.

conexus $1(2018)$ 5-23

(C) 2018 Wolfgang Rother. Dieser Beitrag darf im Rahmen der Lizenz CC BY-NC-ND 4.0 - Creative Commons: Namensnennung/nicht kommerziell/keine Bearbeitungen - weiterverbreitet werden.

\section{(c) (1) 8 (요}

https: / / doi.org/10.24445/ conexus.2018.01.003

Prof. Dr. Wolfgang Rother, Universität Zürich, Philosophisches Seminar, Hirschengraben 56, 8001 Zürich wolfgang.rother@philos.uzh.ch 